\title{
Feasibility of Risk Stratification of Patients Presenting to the Emergency Department With Chest Pain Using HEART Score
}

\author{
Kruti Dhaval Gandhi, MBBS, and Smrati Bajpai Tiwari, MD, DNB, FAIMER
}

Objective: Calculation of HEART score to (1) stratify patients as low-risk, intermediate-risk, high-risk, and to predict the short-term incidence of major adverse cardiovascular events (MACE), and (2) demonstrate feasibility of HEART score in our local settings.

Design: A prospective cohort study of patients with a chief complaint of chest pain concerning for acute coronary syndrome.

Setting: Participants were recruited from the emergency department (ED) of King Edward Memorial Hospital, a tertiary care academic medical center and a resourcelimited setting in Mumbai, India.

Participants: We evaluated 141 patients aged 18 years and older presenting to the ED and stratified them using the HEART score. To assess patients' progress, a follow-up phone call was made within 6 weeks after presentation to the ED.

Measurements: The primary outcomes were a risk stratification, 6-week occurrence of MACE, and performance of unscheduled revascularization or stress testing. The secondary outcomes were discharge or death.

Results: The 141 participants were stratified into low-risk, intermediate-risk, and high-risk groups: 67 (47.52\%),
$44(31.21 \%)$, and $30(21.28 \%)$, respectively. The 6 -week incidence of MACE in each category was $1.49 \%, 18.18 \%$, and $90 \%$, respectively. An acute myocardial infarction was diagnosed in 24 patients (17.02\%), 15 patients (10.64\%) underwent percutaneous coronary intervention ( $\mathrm{PCl})$, and 4 patients (2.84\%) underwent coronary artery bypass graft (CABG). Overall, $98.5 \%$ of low-risk patients and $93.33 \%$ of high-risk patients had an uneventful recovery following discharge; therefore, extrapolation based on results demonstrated reduced health care utilization. All the survey respondents found the HEART score to be feasible. The patient characteristics and risk profile of the patients with and without MACE demonstrated that: patients with MACE were older and had a higher proportion of males, hypertension, type 2 diabetes mellitus, smoking, hypercholesterolemia, prior history of PCI/CABG, and history of stroke.

Conclusion: The HEART score seems to be a useful tool for risk stratification and a reliable predictor of outcomes in chest pain patients and can therefore be used for triage.

Keywords: chest pain; emergency department; HEART score; acute coronary syndrome; major adverse cardiac events; myocardial infarction; revascularization.

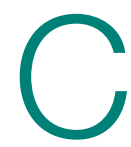
ardiovascular diseases (CVDs), especially coronary heart disease $(\mathrm{CHD})$, have epidemic proportions worldwide. Globally, in 2012, CVD led to 17.5 million deaths, ${ }^{1,2}$ with more than $75 \%$ of them occurring in developing countries. In contrast to developed countries, where mortality from CHD is rapidly declining, it is increasing in developing countries., ${ }^{1,3}$ Current estimates from epidemiologic studies from various parts of India indicate the prevalence of CHD in India to be between $7 \%$ and $13 \%$ in urban populations and $2 \%$ and $7 \%$ in rural populations. ${ }^{4}$

Premature mortality in terms of years of life lost because of CVD in India increased by 59\% over a 20-year span, from 23.2 million in 1990 to 37 million in 2010. ${ }^{5}$ Studies conducted in Mumbai (Mumbai Cohort Study) reported very high CVD mortality rates, approaching 500 per 100000 for men and 250 per 100000 for women.6,7 However, to the best of our knowledge, in the Indian population, there are minimal data on utilization of a triage score, such as the HEART

From the Department of Internal Medicine, Mount Sinai Health System, Icahn School of Medicine at Mount Sinai, New York, NY (Dr. Gandhi), and the School of Medicine, Seth Gordhandas Sunderdas Medical College, and King Edward Memorial Hospital, Mumbai, India (Drs. Gandhi and Tiwari). 
score, in chest pain patients in the emergency department (ED) in a resource-limited setting.

The most common reason for admitting patients to the ED is chest pain. ${ }^{8}$ There are various cardiac and noncardiac etiologies of chest pain presentation. Acute coronary syndrome (ACS) needs to be ruled out first in every patient presenting with chest pain. However, 80\% of patients with ACS have no clear diagnostic features on presentation. ${ }^{9}$ The timely diagnosis and treatment of patients with ACS improves their prognosis. Therefore, clinicians tend to start each patient on ACS treatment to reduce the risk, which often leads to increased costs due to unnecessary, time-consuming diagnostic procedures that may place burdens on both the health care system and the patient. ${ }^{10}$

Several risk-stratifying tools have been developed in the last few years. Both the GRACE and TIMI risk scores have been designed for risk stratification of patients with proven ACS and not for the chest pain population at the ED. ${ }^{11}$ Some of these tools are applicable to patients with all types of chest pain presenting to the ED, such as the Manchester Triage System. Other, more selective systems are devoted to the risk stratification of suspected ACS in the ED. One is the HEART score.12

The first study on the HEART score-an acronym that stands for History, Electrocardiogram, Age, Risk factors, and Troponin-was done by Backus et al, who proved that the HEART score is an easy, quick, and reliable predictor of outcomes in chest pain patients. ${ }^{10}$ The HEART score predicts the short-term incidence of major adverse cardiac events (MACE), which allows clinicians to stratify patients as low-risk, intermediate-risk, and high-risk and to guide their clinical decision-making accordingly. It was developed to provide clinicians with a simple, reliable predictor of cardiac risk on the basis of the lowest score of 0 (very low-risk) up to a score of 10 (very high-risk).

We studied the clinical performance of the HEART score in patients with chest pain, focusing on the efficacy and safety of rapidly identifying patients at risk of MACE. We aimed to determine (1) whether the HEART score is a reliable predictor of outcomes of chest pain patients presenting to the ED; (2) whether the score is feasible in our local settings; and (3) whether it describes the risk profile of patients with and without MACE.

\section{Methods}

\section{Setting}

Participants were recruited from the ED of King Edward Memorial Hospital, a municipal teaching hospital in Mumbai. The study institute is a tertiary care academic medical center located in Parel, Mumbai, Maharashtra, and is a resource-limited setting serving urban, suburban, and rural populations. Participants requiring urgent attention are first seen by a casualty officer and then referred to the emergency ward. Here, the physician on duty evaluates them and decides on admission to the various wards, like the general ward, medical intensive care unit (ICU), coronary care unit (CCU), etc. The specialist's opinion may also be obtained before admission. Critically ill patients are initially admitted to the emergency ward and stabilized before being shifted to other areas of the hospital.

\section{Participants}

Patients aged 18 years and older presenting with symptoms of acute chest pain or suspected ACS were stratified by priority using the chest pain scoring system -the HEART score. Only patients presenting to the ED were eligible for the study. Informed consent from the patient or next of kin was mandatory for participation in the study.

Patients were determined ineligible for the following reasons: a clear cause for chest pain other than ACS (eg, trauma, diagnosed aortic dissection), persisting or recurrent chest pain caused by rheumatic diseases or cancer (a terminal illness), pregnancy, unable or unwilling to provide informed consent, or incomplete data.

\section{Study design}

We conducted a prospective observational study of patients arriving at the tertiary care hospital with a chief complaint of "chest pain" concerning for ACS. All participants provided witnessed written informed consent. Patients were screened over approximately a 3-month period, from July 2019 to October 2019, after acquiring approval from the Institutional Ethics Committee. Any patient who was admitted to the ED due to chest pain, prehospital referrals based on a physician's suspicions of a heart condition, and previous medical treatment due to ischemic heart disease $(\mathrm{HD})$ was eligible. All patients were stratified by priority in our ED using the chest pain scoring system-the HEART 
Table 1. Factors Used to Calculate HEART Score

\begin{tabular}{|c|c|c|c|}
\hline Variable & Score of 0 & Score of 1 & Score of 2 \\
\hline History & $\begin{array}{l}\text { Nonspecific history for ACS, which } \\
\text { is not consistent with chest pain } \\
\text { concerning for ACS }\end{array}$ & $\begin{array}{l}\text { A history that contains mixed } \\
\text { traditional and nontraditional elements } \\
\text { of ACS presentation }\end{array}$ & $\begin{array}{l}\text { A specific history for ACS, with } \\
\text { traditional features of ACS }\end{array}$ \\
\hline ECG & Entirely normal ECG & $\begin{array}{l}\text { Abnormal ECG, with repolarization } \\
\text { abnormalities yet lacking significant } \\
\text { ST-segment depression }\end{array}$ & $\begin{array}{l}\text { Abnormal ECG, with significant } \\
\text { ST-segment deviation } \\
\text { (depression } \pm \text { elevation), either new or } \\
\text { not known to be old (ie, no prior ECG } \\
\text { available for comparison) }\end{array}$ \\
\hline Age, y & $<45$ years & Between 45 and 64 years & $\geq 65$ years \\
\hline Risk factors ${ }^{a}$ & No risk factors & 1 to 2 risk factors & $\begin{array}{l}\geq 3 \text { risk factors or documented } \\
\text { cardiac or systemic atherosclerotic } \\
\text { vascular disease }^{b}\end{array}$ \\
\hline Troponin $^{c}$ & Troponin test negative & NA & Troponin test positive \\
\hline \multicolumn{4}{|c|}{$\begin{array}{l}\text { a Currently treated diabetes mellitus, current or recent (<1 month) smoker, diagnosed hypertension, diagnosed hypercholesterolemia, family history of coronary } \\
\text { artery disease and obesity. } \\
\text { b Peripheral arterial disease, past myocardial infarction, past coronary revascularization procedure, }+/- \text { stroke: results in } 2 \text { points, regardless of the number of } \\
\text { other risk factors } \\
{ }^{c} \text { As is routine for all chest pain patients, the troponin levels are not done in our institute; instead, the positivity and negativity of troponin are done. } \\
\text { ACS, acute coronary syndrome; ECG, electrocardiogram; NA, not applicable. }\end{array}$} \\
\hline
\end{tabular}

score-and were followed up by phone within 6 weeks after presenting to the $\mathrm{ED}$, to assess their progress.

We conducted our study to determine the importance of calculating the HEART score in each patient, which will help to correctly place them into low-, intermediate-, and highrisk groups for clinically important, irreversible adverse cardiac events and guide the clinical decision-making. Patients with low risk will avoid costly tests and hospital admissions, thus decreasing the cost of treatment and ensuring timely discharge from the ED. Patients with high risk will be treated immediately, to possibly prevent a life-threatening, ACS-related incident. Thus, the HEART score will serve as a quick and reliable predictor of outcomes in chest pain patients and help clinicians to make accurate diagnostic and therapeutic choices in uncertain situations.

\section{HEART score}

The total number of points for History, Electrocardiogram (ECG), Age, Risk factors, and Troponin was noted as the HEART score (Table 1).

For this study, the patient's history and ECGs were interpreted by internal medicine attending physicians in the ED. The ECG taken in the emergency room was reviewed and classified, and a copy of the admission ECG was added to the file. The recommendation for patients with a HEART score in a particular range was evaluated. Notably, those with a score of 3 or lower led to a recommendation of reassurance and early discharge. Those with a HEART score in the intermediate range (4-6) were admitted to the hospital for further clinical observation and testing, whereas a high HEART score (7-10) led to admission for intensive monitoring and early intervention. In the analysis of HEART score data, we only used those patients having records for all 5 parameters, excluding patients without an ECG or troponin test.

\section{Results}

Myocardial infarction (MI) was defined based on Universal Definition of Myocardial Infarction. ${ }^{13}$ Coronary revascularization was defined as angioplasty with or without stent placement or coronary artery bypass surgery. ${ }^{14}$ Percutaneous coronary intervention (PCl) was defined as any therapeutic catheter intervention in the coronary arteries. Coronary artery bypass graft (CABG) surgery was defined as any cardiac surgery in which coronary arteries were operated on.

The primary outcomes in this study were the (1) risk stratification of chest pain patients into low-risk, intermediate-risk, and high-risk categories; (2) incidence of a MACE within 6 weeks of initial presentation. MACE consists of acute myocardial infarction (AMI), PCl, CABG, coronary angiography revealing procedurally correctable stenosis managed conservatively, and death due to any cause. 
Our secondary outcomes were discharge or death due to any cause within 6 weeks after presentation.

\section{Follow-up}

Within 6 weeks after presentation to the ED, a follow-up phone call was placed to assess the patient's progress. The follow-up focused on the endpoint of MACE, comprising all-cause death, $\mathrm{MI}$, and revascularization. No patient was lost to follow-up.

\section{Statistical analysis}

We aimed to find a difference in the 6-week MACE between low-, intermediate-, and high-risk categories of the HEART score. The prevalence of CHD in India is $10 \%,{ }^{4}$ and assuming an $\alpha$ of 0.05 , we needed a sample of 141 patients from the ED patient population. Continuous variables were presented by mean (SD), and categorical variables as percentages. We used $t$ test and the Mann-Whitney $U$ test for comparison of means for continuous variables, $\chi^{2}$ for categorical variables, and Fisher's exact test for comparison of the categorical variables. Results with $P<.05$ were considered statistically significant.

We evaluated 141 patients presenting to the ED with chest pain concerning for ACS during the study period, from July 2019 to October 2019. Patients were 57.54 (13.13) years of age. The male to female distribution was 85 to 56 . Other patient characteristics are shown in Table 2.

\section{Primary outcomes}

The risk stratification of the HEART score in chest pain patients and the incidence of 6-week MACE are outlined in Table 3 and Table 4, respectively.

The distribution of the HEART score's 5 elements in the groups with or without MACE endpoints is shown in Table 5. Notice the significant differences between the groups. A follow-up phone call was made within 6 weeks after the presentation to the ED to assess the patient's progress. The 6-week follow-up call data are included in Table 6.

Of 141 patients, 36 patients (25.53\%) were diagnosed with MACE within 6 weeks of presentation. An AMI was diagnosed in 24 patients (17.02\%). Coronary angiography was performed in 31 of 141 patients (21.99\%), 15 patients (10.64\%) underwent $\mathrm{PCl}$, and 4 patients (2.84\%)
Table 2. Patient Characteristics ( $\mathrm{N}=141)$

\begin{tabular}{ll}
\hline Characteristic & No. of patients (\%) \\
\hline Male & $85(60.28)$ \\
\hline Female & $56(39.72)$ \\
\hline Hypertension & $87(61.70)$ \\
\hline Diabetes mellitus & $42(29.79)$ \\
\hline Smoker & $48(34.04)$ \\
\hline Hypercholesterolemia & $56(39.72)$ \\
\hline History of CAD/IHD & $32(22.70)$ \\
\hline History of PCI/CABG & $16(11.34)$ \\
\hline History of stroke & $8(5.67)$ \\
\hline
\end{tabular}

CABG, coronary artery bypass graft; CAD, coronary artery disease; $\mathrm{IHD}$, ischemic heart disease; $\mathrm{PCl}$, percutaneous coronary intervention.

Table 3. HEART Scores of Patients Presenting With Chest Pain to ED ( $\mathrm{N}=141)$

\begin{tabular}{ll}
\hline HEART score & No. of patients (\%) \\
\hline Low-risk (0-3) & $67(47.52)$ \\
\hline Intermediate-risk (4-6) & $44(31.21)$ \\
\hline High-risk (7-10) & $30(21.28)$ \\
\hline
\end{tabular}

Table 4. 6-Week Incidence of MACE (N = 141)

\begin{tabular}{|c|c|c|}
\hline HEART score & MACE, n (\%) & No MACE, n (\%) \\
\hline Low-risk (0-3) $(n=67)$ & $1(1.49)$ & 66 (98.51) \\
\hline Intermediate-risk (4-6) $(n=44)$ & 8 (18.18) & $36(81.82)$ \\
\hline High-risk $(7-10)(n=30)$ & $27(90.00)$ & $3(10.00)$ \\
\hline Total & $36(25.53)$ & $105(74.47)$ \\
\hline
\end{tabular}

a Major adverse cardiovascular events like AMI, revascularization, or death. AMI, acute myocardial infarction; MACE, major adverse cardiovascular events.

underwent CABG. The rest of the patients were treated with medications only.

Myocardial infarction-An AMl was diagnosed in 24 of the 141 patients (17.02\%). Twenty-one of those already had positive markers on admission (apparently, these AMI had started before their arrival to the emergency room). One AMl occurred 2 days after admission in a 66-year-old male, and another occurred 10 days after discharge. A further AMl occurred 2 weeks after discharge. All 3 patients belonged to the intermediate-risk group.

Revascularization-Coronary angiography was performed in 31 of 141 patients (21.99\%). Revascularization was performed in 19 patients (13.48\%), of which 15 were PCls (10.64\%) and 4 were CABGs (2.84\%). 
Table 5. Numerical Distribution of HEART Score's 5 Elements in Groups With or Without MACE (N = 141)

\begin{tabular}{lccccccc}
\hline Elements of HEART score & \multicolumn{3}{c}{ No MACE $(<\mathbf{6}$ weeks) $(\mathbf{n}=\mathbf{1 0 5})$} & \multicolumn{3}{c}{ MACE $(<\mathbf{6}$ weeks) $(\mathbf{n}=\mathbf{3 6})$} \\
\hline & Score of $\mathbf{0}$ & Score of $\mathbf{1}$ & Score of $\mathbf{2}$ & Score of $\mathbf{0}$ & Score of $\mathbf{1}$ & Score of $\mathbf{2}$ & $\boldsymbol{P}$ value \\
\hline History, $\mathrm{n}(\%)$ & $55(52.38)$ & $42(40.00)$ & $8(7.62)$ & $4(11.11)$ & $10(27.78)$ & $22(61.11)$ & $<.001$ \\
\hline ECG, $\mathrm{n}(\%)$ & $69(65.71)$ & $26(24.76)$ & $9(8.57)$ & $7(19.44)$ & $9(25.00)$ & $20(55.55)$ & $<.001$ \\
\hline Age, $\mathrm{n}(\%)$ & $27(25.71)$ & $50(47.62)$ & $28(26.67)$ & $1(2.78)$ & $13(36.11)$ & $22(61.11)$ & $<.001$ \\
\hline Risk factors, $\mathrm{n}(\%)$ & $17(16.19)$ & $50(47.62)$ & $38(36.19)$ & $2(5.56)$ & $10(27.78)$ & $24(66.67)$ & .006 \\
\hline Troponin, $\mathrm{n}(\%)$ & $105(100)$ & NA & NA & $15(41.67)$ & NA & $21(58.33)$ & $<.001$ \\
\hline
\end{tabular}

ECG, electrocardiogram; MACE, major adverse cardiovascular events; NA, not applicable.

Mortality-One patient died from the study population. He was a 72-year-old male who died 14 days after admission. He had a HEART score of 8.

\section{Among the 67 low-risk patients:}

- MACE: Coronary angiography was performed in 1 patient (1.49\%). Among the 67 patients in the low-risk category, there was no cases of AMl or deaths. The remaining 66 patients (98.51\%) had an uneventful recovery following discharge.

- General practitioner (GP) visits/readmissions following discharge: Two of 67 patients (2.99\%) had GP visits following discharge, of which 1 was uneventful. The other patient, a 64-year-old male, was readmitted due to a recurrent history of chest pain and underwent coronary angiography.

\section{Among the 44 intermediate-risk patients:}

- MACE: Of the 7 of 44 patients (15.91\%) who had coronary angiography, 3 patients (6.82\%) had AMl, of which 1 occurred 2 days after admission in a 66-year-old male. Two patients had AMl following discharge. There were no deaths. Overall, 42 of 44 patients (95.55\%) had an uneventful recovery following discharge.

- GP visits/readmissions following discharge: Three of 44 patients (6.82\%) had repeated visits following discharge. One was a GP visit that was uneventful. The remaining 2 patients were diagnosed with $\mathrm{AMl}$ and readmitted following discharge. One AMl occurred 10 days after discharge in a patient with a HEART score of 6; another occurred 2 weeks after discharge in a patient with a HEART score of 5 .

\section{Among the 30 high-risk patients:}

- MACE: Twenty-three of 30 patients (76.67\%) underwent coronary angiography. One patient died 5 days after discharge. The patient had a HEART score of 8. Most patients however, had an uneventful recovery following discharge (28, 93.33\%).

- GP visits/readmissions following discharge: Five of 30 patients (16.67\%) had repeated visits following discharge. Two were uneventful. Two patients had a history of recurrent chest pain that resolved on Sorbitrate. One patient was readmitted 2 weeks following discharge due to a complication: a left ventricular clot was found. The patient had a HEART score of 10.

Secondary outcome-Overall, 140 of 141 patients were discharged. One patient died: a 72-year-old male with a HEART score of 8.

Feasibility-To determine the ease and feasibility of performing a HEART score in chest pain patients presenting to the ED, a survey was distributed to the internal medicine physicians in the ED. In the survey, the Likert scale was used to rate the ease of utilizing the HEART score and whether the physicians found it feasible to use it for risk stratification of their chest pain patients. A total of 12 of 15 respondents (80\%) found it "easy" to use. Of the remaining 3 respondents, 2 (13.33\%) rated the HEART score "very easy" to use, while 1 (6.66\%) considered it "difficult" to work with. None of the respondents said that it was not feasible to perform a HEART score in the ED.

\section{Risk factors for reaching an endpoint:}

We compared risk profiles between the patient groups with and without an endpoint. The group of patients with 
Table 6. 6-Week Follow-up Phone Call Data

\begin{tabular}{|c|c|c|c|}
\hline Follow-up data & Low-risk $(n=67)$ & Intermediate-risk $(n=44)$ & High-risk $(n=30)$ \\
\hline & \multicolumn{3}{|c|}{ HEART score } \\
\hline MACE within 6 weeks: 36/141 & 1 & 8 & 27 \\
\hline AMl & 0 & 3 & 21 \\
\hline Coronary angiography & 1 & 7 & 23 \\
\hline $\mathrm{PCl}$ & 0 & 4 & 11 \\
\hline CABG & 0 & 0 & 4 \\
\hline Death & 0 & 0 & 1 \\
\hline GP visits and/or readmissions following discharge & 2 & $3^{\mathrm{a}}$ & $5^{\mathrm{b}}$ \\
\hline
\end{tabular}

MACE were older and had a higher proportion of males than the group of patients without MACE. Moreover, they also had a higher prevalence of hypertension, type 2 diabetes mellitus, smoking, hypercholesterolemia, prior history of PCl/CABG, and history of stroke. These also showed a significant association with MACE. Obesity was not included in our risk factors as we did not have data collected to measure body mass index. Results are represented in Table 7.

\section{Discussion}

Our study described a patient population presenting to an ED with chest pain as their primary complaint. The results of this prospective study confirm that the HEART score is an excellent system to triage chest pain patients. It provides the clinician with a reliable predictor of the outcome (MACE) after the patient's arrival, based on available clinical data and in a resource-limited setting like ours.

Cardiovascular epidemiology studies indicate that this has become a significant public health problem in India. ${ }^{1}$ Several risk scores for ACS have been published in European and American guidelines. However, in the Indian population, minimal data are available on utilization of such a triage score (HEART score) in chest pain patients in the ED in a resource-limited setting, to the best of our knowledge. In India, only 1 such study is reported, ${ }^{15}$ at the Sundaram Medical Foundation, a 170-bed community hospital in Chennai. In this study, 13 of 14 patients (92.86\%) with a high HEART score had MACE, indicating a sensitivity of $92.86 \%$; in the 44 patients with a low HEART score, 1 patient (2.22\%) had MACE, indicating a specificity of $97.78 \%$; and in the 28 patients with a moderate HEART score, 12 patients (42.86\%) had MACE.

In looking for the optimal risk-stratifying system for chest pain patients, we analyzed the HEART score. The first study on the HEART score was done Backus et al, proving that the HEART score is an easy, quick, and reliable predictor of outcomes in chest pain patients..$^{10}$ The HEART score had good discriminatory power, too. The C statistic for the HEART score for ACS occurrence shows a value of 0.83 . This signifies a good-to-excellent ability to stratify all-cause chest pain patients in the ED for their risk of MACE. The application of the HEART score to our patient population demonstrated that the majority of the patients belonged to the low-risk category, as reported in the first cohort study that applied the HEART score. ${ }^{8}$ The relationship between the HEART score category and occurrence of MACE within 6 weeks showed a curve with 3 different patterns, corresponding to the 3 risk categories defined in the literature. ${ }^{11,12}$ The risk stratification of chest pain patients using the 3 categories (0-3, 4-6, 7-10) identified MACE with an incidence similar to the multicenter study of Backus et al, ${ }^{10,11}$ but with a greater risk of MACE in the high-risk category (Figure).

Thus, our study confirmed the utility of the HEART score categories to predict the 6-week incidence of MACE. The sensitivity, specificity, and positive and negative predictive values for the established cut-off scores of 4 and 7 are shown in Table 8. The patients in the low-risk category, corresponding to a score $<4$, had a very high negative 
Table 7. Risk Profile of Patients With and Without MACE (N = 141)

\begin{tabular}{lccc}
\hline & MACE $(\mathbf{n}=\mathbf{3 6})$ & No MACE $(\mathbf{n}=\mathbf{1 0 5})$ & $\boldsymbol{P}$ value \\
\hline Mean age (SD), $\mathrm{y}$ & $67.972(8.882)$ & $54.152(12.745)$ & $t$ test $-P<.001$ \\
\hline Male, $\mathrm{n}(\%)$ & $30(83.33)$ & $55(52.38)$ & .001 \\
\hline Hypertension, $\mathrm{n}(\%)$ & $29(80.56)$ & $58(55.24)$ & .007 \\
\hline Diabetes mellitus, $\mathrm{n}(\%)$ & $19(52.78)$ & $23(21.90)$ & .005 \\
\hline Smoker, $\mathrm{n}(\%)$ & $20(55.55)$ & $28(26.67)$ & .02 \\
\hline Hypercholesterolemia, $\mathrm{n}(\%)$ & $24(66.67)$ & $32(30.48)$ & $<.001$ \\
\hline History of CAD/IHD, $\mathrm{n}(\%)$ & $15(41.67)$ & $17(16.19)$ & .002 \\
\hline History of PCI/CABG, $\mathrm{n}(\%)$ & $9(25.00)$ & $7(6.67)$ & .003 \\
\hline History of stroke, $\mathrm{n}(\%)$ & $5(13.89)$ & $3(2.85)$ & .01 \\
\hline
\end{tabular}

CABG, coronary artery bypass graft; CAD, coronary artery disease; IHD, ischemic heart disease; MACE, major adverse cardiovascular events; $\mathrm{PCl}$, percutaneous coronary intervention.

predictive value, thus identifying a small-risk population. The patients in the high-risk category (score $\geq 7$ ) showed a high positive predictive value, allowing the identification of a high-risk population, even in patients with more atypical presentations. Therefore, the HEART score may help clinicians to make accurate management choices by being a strong predictor of both event-free survival and potentially life-threatening cardiac events. ${ }^{11,12}$

Our study tested the efficacy of the HEART score pathway in helping clinicians make smart diagnostic and therapeutic choices. It confirmed that the HEART score was accurate in predicting the short-term incidence of MACE, thus stratifying patients according to their risk severity. In our study, 67 of 141 patients (47.52\%) had low-risk HEART scores, and we found the 6-week incidence of MACE to be $1.49 \%$. We omitted the diagnostic and treatment evaluation for patients in the low-risk category and moved onto discharge. Overall, 66 of 67 patients (98.51\%) in the low-risk category had an uneventful recovery following discharge. Only 2 of 67 these patients (2.99\%) of patients had health care utilization following discharge. Therefore, extrapolation based on results demonstrates reduced health care utilization. Previous studies have shown similar results. ${ }^{9,12,14,16}$ For instance, in a prospective study conducted in the Netherlands, low-risk patients representing $36.4 \%$ of the total were found to have a Iow MACE rate $(1.7 \%){ }^{9}$ These low-risk patients were categorized as appropriate and safe for ED discharge without additional cardiac evaluation or inpatient admission. ${ }^{9}$ Another retrospective study in Portugal, ${ }^{12}$ and one in
Chennai, India, ${ }^{15}$ found the 6-week incidence of MACE to be $2.00 \%$ and $2.22 \%$, respectively. The results of the first HEART Pathway Randomized Control Trial ${ }^{14}$ showed that the HEART score pathway reduces health care utilization (cardiac testing, hospitalization, and hospital length of stay). The study also showed that these gains occurred without any of the patients that were identified for early discharge, suffering from MACE at 30 days, or secondary increase in cardiac-related hospitalizations. Similar results were obtained by a randomized trial conducted in North Carolina ${ }^{17}$ that also demonstrated a reduction in objective cardiac testing, a doubling of the rate of early discharge from the ED, and a reduced length of stay by half a day. Another study using a modified HEART score also demonstrated that when low-risk patients are evaluated with cardiac testing, the likelihood for false positives is high. ${ }^{16}$ Hoffman et al also reported that patients randomized to coronary computed tomographic angiography (CCTA) received $>2.5$ times more radiation exposure. ${ }^{16}$ Thus, low-risk patients may be safely discharged without the need for stress testing or CCTA.

In our study, 30 out of 141 patients (21.28\%) had high-risk HEART scores (7-10), and we found the 6-week incidence of MACE to be $90 \%$. Based on the pathway leading to inpatient admission and intensive treatment, 23 of 30 patients $(76.67 \%)$ patients in our study underwent coronary angiography and further therapeutic treatment. In the high-risk category, 28 of 30 patients (93.33\%) patients had an uneventful recovery following discharge. Previous studies have shown similar results. A retrospective study 
Table 8. Sensitivity, Specificity, Positive, and Negative Predictive Values for 6-Week Incidence of MACE Using the HEART Score

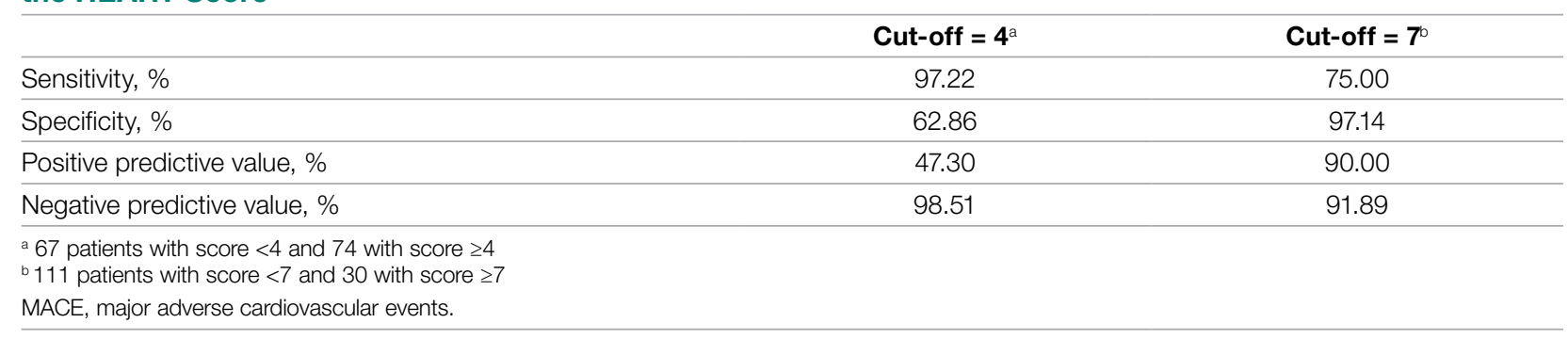

in Portugal showed that $76.9 \%$ of the high-risk patients had a 6-week incidence of MACE. ${ }^{22}$ In a study in the Netherlands, ${ }^{9} 72.7 \%$ of high-risk patients had a 6 -week incidence of MACE. Therefore, a HEART score of $\geq 7$ in patients implies early aggressive treatment, including invasive strategies, when necessary, without noninvasive treatment preceding it. ${ }^{8}$

In terms of intermediate risk, in our study 44 of 141 patients (31.21\%) patients had an intermediate-risk HEART score (4-6), and we found the 6-week incidence of MACE to be $18.18 \%$. Based on the pathway, they were kept in the observation ward on admission. In our study, 7 of 44 patients (15.91\%) underwent coronary angiography and further treatment; 42 of 44 patients (95.55\%) had an uneventful recovery following discharge. In a prospective study in the Netherlands, $46.1 \%$ of patients with an intermediate score had a 6-week MACE incidence of $16.6 \% .^{10}$ Similarly, in another retrospective study in Portugal, the incidence of 6 -week MACE in intermediate-risk patients (36.7\%) was found to be $15.6 \% .^{12}$ Therefore, in patients with a HEART score of 4-6 points, immediate discharge is not an option, as this figure indicates a risk of $18.18 \%$ for an adverse outcome. These patients should be admitted for clinical observation, treated as an ACS awaiting final diagnosis, and subjected to noninvasive investigations, such as repeated troponin. Using the HEART score as guidance in the treatment of chest pain patients will benefit patients on both sides of the spectrum. ${ }^{11,12}$

Our sample presented a male predominance, a wide range of age, and a mean age similar to that of previous studies. ${ }^{12.16}$ Some risk factors, we found, can increase significantly the odds of chest pain being of cardiovascular origin, such as male gender, smoking, hypertension, type 2 diabetes mellitus, and hypercholesterolemia. Other studies also reported similar findings. 8,12,16 Risk factors for premature CHD have been quantified in the case-control INTERHEART study. ${ }^{1}$ In the INTERHEART study, 8 common risk factors explained $>90 \%$ of AMls in South Asian and Indian patients. The risk factors include dyslipidemia, smoking or tobacco use, known hypertension, known diabetes, abdominal obesity, physical inactivity, low fruit and vegetable intake, and psychosocial stress. ${ }^{1}$ Regarding the feasibility of treating physicians using the HEART score in the ED, we observed that, based on the Likert scale, $80 \%$ of survey respondents found it easy to use, and $100 \%$ found it feasible in the ED.

However, there were certain limitations to our study. It involved a single academic medical center and a small sample size, which limit generalizability of the findings. In addition, troponin levels are not calculated at our institution, as it is a resource-limited setting; therefore, we used a positive and negative as +2 and 0 , respectively.

\section{Conclusion}

The HEART score provides the clinician with a quick and reliable predictor of outcome of patients with chest pain after arrival to the ED and can be used for triage. For patients with low HEART scores (0-3), short-term MACE can be excluded with greater than $98 \%$ certainty. In these patients, one may consider reserved treatment and discharge policies that may also reduce health care utilization. In patients with high HEART scores (7-10), the high risk of MACE (90\%) may indicate early aggressive treatment, including invasive strategies, when necessary. Therefore, 


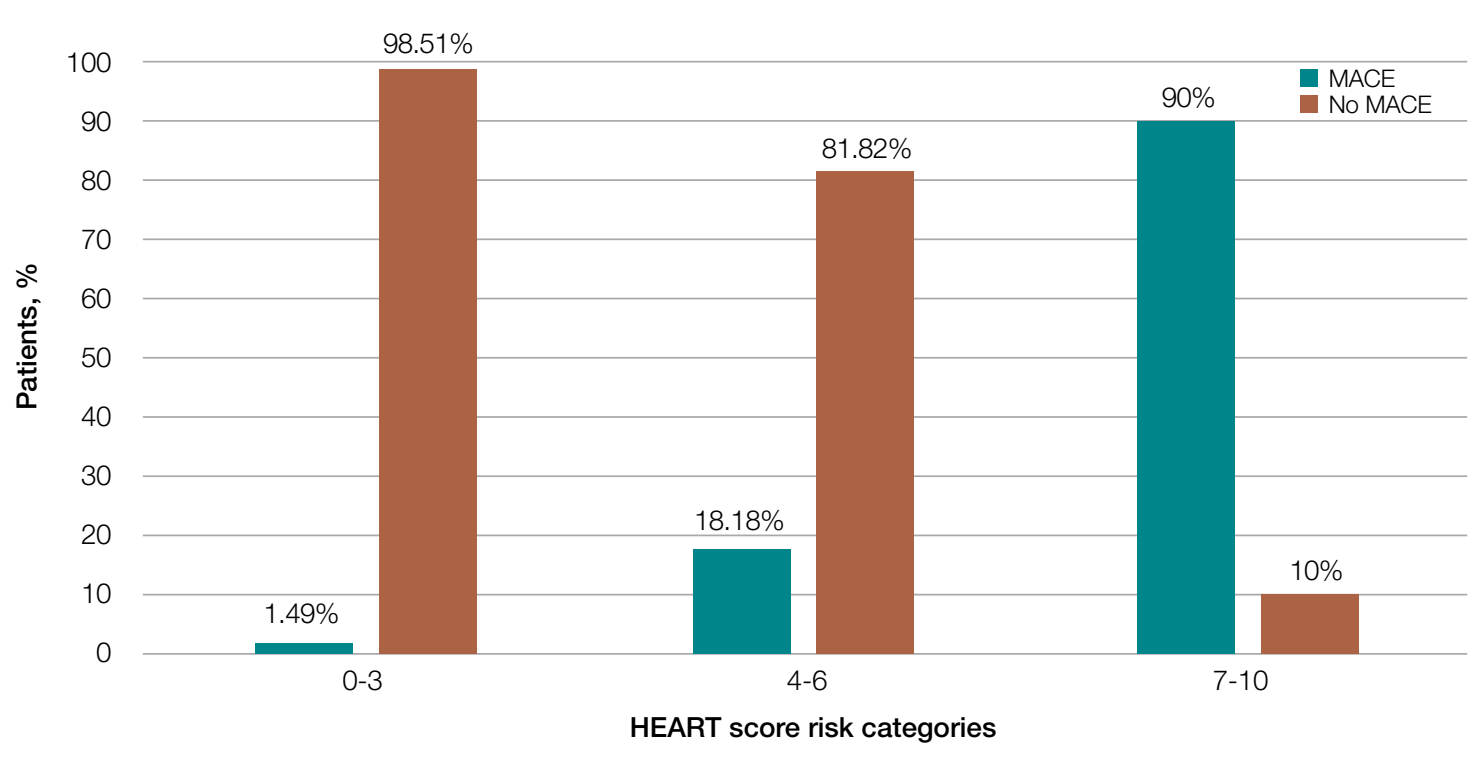

Figure. Six-week incidence of MACE in each HEART score category. MACE indicates major adverse cardiovascular events.

the HEART score may help clinicians make accurate management choices by being a strong predictor of both eventfree survival and potentially life-threatening cardiac events. Age, gender, and cardiovascular risk factors may also be considered in the assessment of patients. This study confirmed the utility of the HEART score categories to predict the 6-week incidence of MACE.

Corresponding author: Smrati Bajpai Tiwari, MD, DNB, FAIMER, Department of Medicine, Seth Gordhandas Sunderdas Medical College and King Edward Memorial Hospital, Acharya Donde Marg, Parel, Mumbai 400 012, Maharashtra, India; smrati.bajpai@ gmail.com.

Financial disclosures: None.

doi:10.12788/jcom.0059

\section{References}

1. Gupta R, Mohan I, Narula J. Trends in coronary heart disease epidemiology in India. Ann Glob Health. 2016;82:307-315.

2. World Health Organization. Global status report on noncommunicable diseases 2014. Accessed June 22, 2021. https:// apps.who.int/iris/bitstream/handle/10665/148114/9789241564854_ eng.pdf

3. Fuster V, Kelly BB, eds. Promoting Cardiovascular Health in the Developing World: A Critical Challenge to Achieve Global Health. Institutes of Medicine; 2010.

4. Krishnan MN. Coronary heart disease and risk factors in India-on the brink of an epidemic. Indian Heart J. 2012;64:364-367.
5. Prabhakaran D, Jeemon P, Roy A. Cardiovascular diseases in India: current epidemiology and future directions. Circulation. 2016;133:1605-1620.

6. Aeri B, Chauhan $\mathrm{S}$. The rising incidence of cardiovascular diseases in India: assessing its economic impact. J Prev Cardiol. 2015;4:735-740.

7. Pednekar M, Gupta R, Gupta PC. Iliteracy, low educational status and cardiovascular mortality in India. BMC Public Health. 2011;11:567.

8. Six AJ, Backus BE, Kelder JC. Chest pain in the emergency room: value of the HEART score. Neth Heart J. 2008;16:191-196.

9. Backus BE, Six AJ, Kelder JC, et al. A prospective validation of the HEART score for chest pain patients at the emergency department. Int J Cardiol. 2013;168;2153-2158.

10. Backus BE, Six AJ, Kelder JC, et al. Chest pain in the emergency room: a multicenter validation of the HEART score. Crit Pathw Cardiol. 2010;9:164-169.

11. Backus BE, Six AJ, Kelder JH, et al. Risk scores for patients with chest pain: evaluation in the emergency department. Curr Cardiol Rev. 2011;7:2-8.

12. Leite L, Baptista R, Leitão J, et al. Chest pain in the emergency department: risk stratification with Manchester triage system and HEART score. BMC Cardiovasc Disord. 2015;15:48.

13. Thygesen K, Alpert JS, Jaffe AS, et al. Fourth Universal Definition of Myocardial Infarction. Circulation. 2018;138:e618-e651.

14. Mahler SA, Riley RF, Hiestand BC, et al. The HEART Pathway randomized trial: identifying emergency department patients with acute chest pain for early discharge. Circ Cardiovasc Qual Outcomes. 2015;8:195-203.

15. Natarajan B, Mallick $P$, Thangalvadi TA, Rajavelu P. Validation of the HEART score in Indian population. Int J Emerg Med. 2015,8(suppl 1):P5.

16. McCord J, Cabrera R, Lindahl B, et al. Prognostic utility of a modified HEART score in chest pain patients in the emergency department. Circ Cardiovasc Qual Outcomes. 2017;10:e003101.

17. Mahler SA, Miller CD, Hollander JE, et al. Identifying patients for early discharge: performance of decision rules among patients with acute chest pain. Int J Cardiol. 2012;168:795-802. 